\title{
CHARACTERIZING EXPOSURE-DISEASE ASSOCIATION IN HUMAN POPULATIONS USING THE LORENZ CURVE AND GINI INDEX
}

\author{
WEN-CHUNG LEE \\ Graduate Institute of Epidemiology, College of Public Health, National Taiwan University, Taipei, Taiwan, R.O.C.
}

\begin{abstract}
SUMMARY
To characterize exposure-disease association in human populations, epidemiologists have long relied upon such indices as 'relative risk' and/or 'attributable risk'. However, the relative risk is not in a common unit which permits comparison across different exposures or different diseases and the attributable risk may not adequately catch and describe the variation of disease risks in populations. The present paper discusses the possibility of using the summary index of the Lorenz curve, the Gini index, as an alternative measure of exposure-disease association. It is found that this index can be interpreted in several ways (as the coefficient of deviation in disease risk or relative risk, the information content of the exposure, the impact fraction of an exposure-lowering programme, and the averaged impact fraction) and is a promising alternative as a fundamental measure in epidemiology. Further studies are warranted to investigate its statistical properties. (C) 1997 by John Wiley \& Sons, Ltd.
\end{abstract}

\section{INTRODUCTION}

Describing how various diseases occur in human populations is a fundamental issue in epidemiology. The occurrence of a particular disease in a population often varies remarkably according to a wide range of factors or 'exposures', such as sex, age, ethnicity, social class, smoking status and drinking behaviour. Therefore, quantitative measures which can adequately catch and describe such variation of disease risk are of much importance. Based on these measures of exposure-disease association, it is then possible to make epidemiological inferences about disease aetiology or to set priorities for intervention.

To characterize the relationship between an exposure and a disease, by far the most widely used index has been the 'relative risk'. Experience shows that such an index provides a stable measure of association in a wide variety of human populations and hence may be viewed as a 'biological constant'. ${ }^{1}$ It also possesses some logical properties which are desirable for causal inferences. ${ }^{2}$ For a continuous or a polytomous exposure, we can also tailor the relative risks to represent the dose-response gradient. ${ }^{3}$ However, it is noted that when we wish to compare the strengths of association between several exposures and a particular disease, the relative risk has some intrinsic difficulties. For example, consider the association between cardiovascular disease and three exposures (risk factors): cholesterol level (continuous scale); smoking habits (polytomous scale with three levels of 'no smoking', 'moderate smoking' and 'heavy smoking'); and educational status (polytomous scale with, say, five levels). These exposures are of disparate measurement scales and hence, even if the relative risks in different exposure levels for these factors are given, we 
may still have difficulty in deciding which one is the 'strongest' risk factor for cardiovascular disease. Similar difficulty also arises when we wish to compare the impact of a particular exposure (say, alcohol drinking, with three levels of none/moderate/heavy) upon different diseases (say, liver cancer and congenital anomalies). The relative risks for liver cancer may be something like $1 \cdot 0,3 \cdot 0$ and $8 \cdot 0$, and those for congenital anomalies may be, for example, $1 \cdot 0,2 \cdot 0$ and $9 \cdot 0$. It is clear that for moderate drinkers the impact of the exposure is larger for liver cancer than for congenital anomalies, while the reverse is true for the heavy drinkers, but it is difficult to judge, given the relative risks, which disease is influenced more as a whole by drinking behaviour. This problem of incomparability becomes more severe if the exposure has more levels or is a continuous scale. It seems that, for comparison of the magnitude of exposure-disease association across different exposures and/or across different diseases, a summary index (of the relative risks) needs to be constructed.

In epidemiology, the 'attributable risk'1,3,4 (also called the 'population attributable risk per cent' ${ }^{5}$ or the 'aetiologic fraction' ${ }^{6}$ ) is another commonly used index aimed at measuring the public health impact of an exposure on disease burden. It was first introduced by Levin ${ }^{7}$ for the simple situation of binary exposures (presence/absence), being the proportion of cases expected to be eliminated by removal of the exposure. Later the concept was generalized to polytomous or even continuous risk factors. In such situations, the index (now referred to as the 'generalized impact fraction') still possesses the same interpretation of the proportion of cases expected to be eliminated but with the reduction of high levels of exposure to some 'target level' instead of complete removal. ${ }^{8}$ From the formula of the attributable risk and/or the generalized impact fraction, we can see that each of them has, in effect, summarized the relative risks into one single-valued index which is between 0 and $1 .^{9,10}$ Therefore, the magnitudes of the impacts upon a particular disease of two exposures with disparate measurement scales and/or of the impacts upon two different diseases of a particular exposure can be compared, at least literally.

In this paper, it will be shown that though the attributable risk (or the generalized impact fraction) has some public health implications, it may not adequately catch and describe the variation of disease risks in populations. To this end, we apply the summary index of the 'Lorenz curve', the 'Gini index', as an alternative. The Lorenz curve and the Gini index have been widely used by economists to assess the distributional properties of family income and wealth ${ }^{11}$ and by demographers to quantify the degree of population concentration. ${ }^{12}$ Recently, the technique has been applied to the analysis of seasonal data in detecting and testing for temporal clustering of disease occurrences. ${ }^{13}$ The possibility of using the Lorenz curve and Gini index to characterize exposure-disease association in human populations has not yet been explored, however.

\section{INADEQUACY OF THE ATTRIBUTABLE RISK}

We present hypothetical examples in Tables I to III to demonstrate that using the index of attributable risk may fail to catch the inherent nature of risk variation in a population. Table I concerns the risk of a hypothetical disease $\mathrm{X}$ in a hypothetical population $\mathrm{A}$. The risk of disease $\mathrm{X}$ (probability of disease $\mathrm{X}$ over a certain period of time) is assumed to vary according to a particular polytomous exposure with five levels (none/light/medium/heavy/extra-heavy). The risks and the relative risks (the lowest level of 'none' is taken as the reference level) in the various exposure levels are shown in Table I. Also shown are the generalized impact fractions for each exposure level defined in this paper as the proportion of cases expected to be eliminated if those with heavier exposure in the population were exposed to the indicated level instead. The generalized impact fraction for the lowest exposure level, 'none', by definition is the proportion of cases eliminated if the exposure is completely removed and hence is identical to the traditionally defined attributable risk. For this example, the figure is $67 \cdot 1$ per cent. 
Table I. Risk of disease $\mathrm{X}$ in population A

\begin{tabular}{lrrrrr}
\hline Characteristics of the population & \multicolumn{5}{c}{ Exposure status } \\
\cline { 2 - 6 } & None & Light & Medium & Heavy & Extra-heavy \\
\hline Case number & 20 & 60 & 184 & 24 & 16 \\
Population number & 20,000 & 30,000 & 46,000 & 3,000 & 1,000 \\
Exposure prevalence (\%) & 20 & 30 & 46 & 3 & 1 \\
Risk of disease & $0 \cdot 001$ & $0 \cdot 002$ & $0 \cdot 004$ & $0 \cdot 008$ & $0 \cdot 016$ \\
Relative risk & $1 \cdot 0$ & $2 \cdot 0$ & $4 \cdot 0$ & $8 \cdot 0$ & $16 \cdot 0$ \\
Generalized impact fraction (\%)* & $67 \cdot 1$ & $40 \cdot 8$ & $7 \cdot 9$ & $2 \cdot 6$ & $0 \cdot 0$ \\
\hline
\end{tabular}

* Proportion of cases expected to be eliminated if those with heavier exposure in the population were exposed to the indicated level instead. The generalized impact fraction for the lowest exposure level ('none') is identical to the traditionally defined 'attributable risk'

Table II. Risk of disease $\mathrm{X}$ in population $\mathrm{B}$

\begin{tabular}{lrrrrr}
\hline Characteristics of the population & \multicolumn{5}{c}{ Exposure status } \\
\cline { 2 - 6 } & None & Light & Medium & Heavy & Extra-heavy \\
\hline Case number & 40 & 240 & 56 & 144 & 128 \\
Population number & 20,000 & 60,000 & 7,000 & 9,000 & 4,000 \\
Exposure prevalence (\%) & 20 & 60 & 7 & 9 & 4 \\
Risk of disease & $0 \cdot 002$ & $0 \cdot 004$ & $0 \cdot 008$ & $0 \cdot 016$ & $0 \cdot 032$ \\
Relative risk & $1 \cdot 0$ & $2 \cdot 0$ & $4 \cdot 0$ & $8 \cdot 0$ & $16 \cdot 0$ \\
Generalized impact fraction (\%)* & $67 \cdot 1$ & $40 \cdot 8$ & $27 \cdot 6$ & $10 \cdot 5$ & $0 \cdot 0$ \\
\hline
\end{tabular}

* Proportion of cases expected to be eliminated if those with heavier exposure in the population were exposed to the indicated level instead. The generalized impact fraction for the lowest exposure level ('none') is identical to the traditionally defined 'attributable risk'

Now turn to Table II where the risk for the same disease $\mathrm{X}$ in another population $\mathrm{B}$ is described. The exposure under concern is the same as in Table I but now with different exposure prevalences in population B. The 'relative risks' in the various exposure levels for population B are assumed identical to those in population A (note that the 'risks' themselves are not the same in the two populations). This assumption is reasonable since empirical evidence suggests that the relative risks are biological constants and are independent of the populations from which they are derived. ${ }^{1}$ After simple calculation, we find that the attributable risk in population B is also $67 \cdot 1$ per cent, from which we get an impression that the public health impact of the exposure on disease burden is the same in the two populations. However, this is not the case. First, from the generalized impact fractions, we see that if an intervention programme sets a goal not of completely removing the exposure, which may be impossible, but at reducing it to no heavier than the 'medium' level, the proportion of cases expected to be eliminated (the impact) for this more 'realistic' programme is much larger for population B (27.6 per cent) than for population A ( 7.9 per cent). Secondly, we see that although the percentage of the exposed population (regardless of the exposure levels) is the same for the two populations ( 30 per cent +46 per cent +3 per cent +1 per cent $=60$ per cent +7 per cent +9 per cent +4 per cent $=80$ per cent), population A is actually less heavily exposed (those exposed to 'extra-heavy' and 'heavy' only constitute 1 per 
Table III. Risk of disease Y in population A

\begin{tabular}{lrrrrr}
\hline Characteristics of the population & \multicolumn{5}{c}{ Exposure status } \\
\cline { 2 - 6 } & None & Light & Medium & Heavy & Extra-heavy \\
\hline Case number & 100 & 510 & 828 & 60 & 22 \\
Population number & 20,000 & 30,000 & 46,000 & 3,000 & 1,000 \\
Exposure prevalence (\%) & 20 & 30 & 46 & 3 & 1 \\
Risk of disease & $0 \cdot 005$ & $0 \cdot 017$ & $0 \cdot 018$ & $0 \cdot 020$ & $0 \cdot 022$ \\
Relative risk & $1 \cdot 0$ & $3 \cdot 4$ & $3 \cdot 6$ & $4 \cdot 0$ & $4 \cdot 4$ \\
Generalized impact fraction (\%)* & $67 \cdot 1$ & $3 \cdot 9$ & $0 \cdot 7$ & $0 \cdot 1$ & $0 \cdot 0$ \\
\hline
\end{tabular}

* Proportion of cases expected to be eliminated if those with heavier exposure in the population were exposed to the indicated level instead. The generalized impact fraction for the lowest exposure level ('none') is identical to the traditionally defined 'attributable risk'

cent and 3 per cent, respectively, for population $\mathrm{A}$, whereas the corresponding figures for population B are 4 per cent and 9 per cent, respectively). It seems that the index of the attributable risk alone may not be adequate in characterizing the exposure impact.

Consider now the example in Table III, where the risk for another disease $\mathrm{Y}$ in population A is depicted. The exposure under concern is the same one as in Table I. From the relative risks for different exposure levels, we can see that the dose-response gradient the exposure exerts for disease Y (Table III) is not as striking as for disease X (Table I). The risk for the highest exposure level is only 4.4 times that of the lowest level for disease $\mathrm{Y}$, whereas the figure is $16 \cdot 0$ for disease $\mathrm{X}$. Naturally, we will agree that the impact of the particular exposure upon disease $X$ is greater than that upon disease Y. Unfortunately, using the index of attributable risk alone cannot reveal this simple fact either (the attributable risks are $67 \cdot 1$ per cent for both diseases).

From these hypothetical examples, we feel that the traditionally defined attributable risk, though it is interpretable, may not be realistic for an actual intervention programme. Furthermore, from the point of view of describing disease variation in human populations, it often fails to catch the important variability in risk. Using the generalized impact fractions may ease some of the problems, provided that different impact fractions for different 'target levels' are presented. However, we are then left with the same trouble with the relative risks in that a direct comparison of the impacts between different exposures and/or between different diseases is again not possible.

In the next section, we introduce the Lorenz curve and the Gini index to characterize exposure-disease association in human populations. The same examples in Tables I to III will be used to demonstrate the advantages of the new method.

\section{LORENZ CURVE AND GINI INDEX}

The data in Table I are used to demonstrate the construction of the Lorenz curve and the calculation of the Gini index. For this example, the Lorenz curve is simply the plot of the cumulative percentage of cases against the cumulative percentage of population (see Figure 1). If the risks of disease are not monotonically increasing as the exposure becomes heavier, we have to re-arrange the data from the lowest to highest risk before the calculation of the cumulative percentages. (Note that our usage of the Lorenz curve is somewhat different from that of economists or demographers. Economists, in assessing the distributional properties of family income and wealth, often plot the cumulative percentage of 'income' against the cumulative 


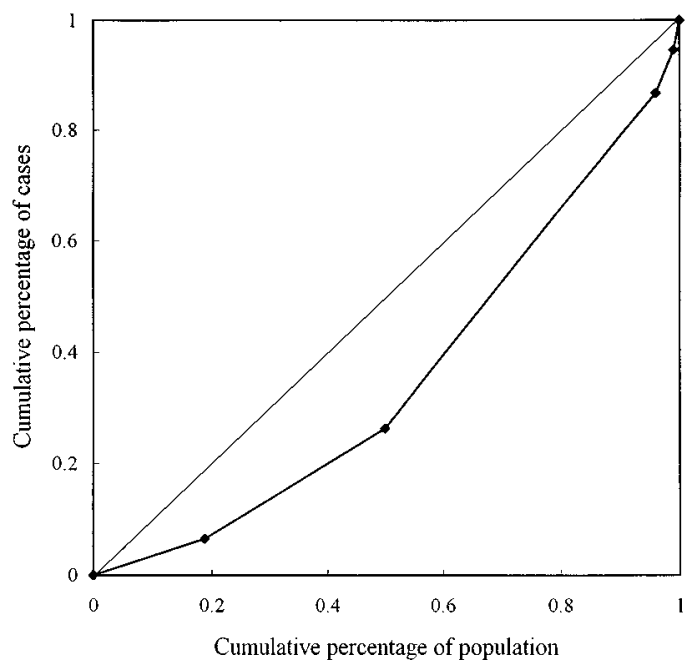

Figure 1. The Lorenz curve for the example in Table I. The Gini index is twice the area between the Lorenz curve and the diagonal line

percentage of 'population', while for the purpose of quantifying the degree of population concentration, demographers plot the cumulative percentage of 'population number' against the cumulative percentage of 'land area'.). It is clear that the Lorenz curve would strictly follow the diagonal line, in the case when the risk of disease is exactly equal in different exposure levels (in other words, the exposure is not a risk factor). If all the cases could only occur in one particular exposure level, the Lorenz curve would coincide with the $X$-axis throughout except for the last exposure level where the curve jumps to the right uppermost point $(1,1)$. This observation suggests the use of the 'area between the Lorenz curve and the diagonal' as the index of exposure-disease association. A larger area, resulting from a more bowed Lorenz curve, means that the risk of disease is more variable in the population, while a smaller area or a flatter curve indicates a more uniform distribution in disease risk.

The Gini index is defined as twice the area between the Lorenz curve and the diagonal line, or equivalently as the ratio of the aforementioned area to the area of the triangle below the diagonal line. Clearly this index is between zero and one, with larger values indicating greater variability while smaller ones indicate greater uniformity. Calculating the index is straightforward using simple algebra. For the examples in Tables I to III, the Gini indices are 0.294, 0.408 and 0.149, respectively (see Table IV).

\section{INTERPRETATION OF THE GINI INDEX}

It is of interest to note that such a geometrically defined Gini index can be interpreted in several ways. All suggest that such an index is ideal for the purpose of characterizing exposure-disease association in human populations. These are explained as follows:

(i) 'Coefficient of deviation' in disease risk. To measure the variation of risk in a population, using an index as 'coefficient of variation' (standard deviation/mean) seems better than simply the variance or the standard deviation. This is because the coefficient of variation produces a measure of relative variation - variation that is relative to the size of the mean 
Table IV. Various indices calculated from the data in Tables I to III

\begin{tabular}{|c|c|c|c|}
\hline \multirow[t]{2}{*}{ Indices } & \multicolumn{3}{|c|}{ Examples in } \\
\hline & Table I & Table II & Table III \\
\hline Gini index* & $0 \cdot 294$ & $0 \cdot 408$ & $0 \cdot 149$ \\
\hline Averaged disease risk $^{\dagger}$ & $0 \cdot 00304$ & $0 \cdot 00608$ & 0.0152 \\
\hline Mean difference in disease risk ${ }^{\ddagger}$ & $0 \cdot 00179$ & $0 \cdot 00496$ & $0 \cdot 00453$ \\
\hline Averaged relative risk ${ }^{\dagger}$ & $3 \cdot 04$ & $3 \cdot 04$ & $3 \cdot 04$ \\
\hline Mean difference in relative risk $^{\ddagger}$ & $1 \cdot 79$ & $2 \cdot 48$ & $0 \cdot 907$ \\
\hline 'Information content' of the exposure & $0 \cdot 647$ & $0 \cdot 704$ & $0 \cdot 575$ \\
\hline $\begin{array}{l}\text { Impact fraction of an 'exposure-lowering' } \\
\text { programme }(\%)^{\|}\end{array}$ & $29 \cdot 4$ & $40 \cdot 8$ & $14 \cdot 9$ \\
\hline Averaged impact fraction $(\%)^{\dagger}$ & $29 \cdot 4$ & $40 \cdot 8$ & $14 \cdot 9$ \\
\hline \multicolumn{4}{|c|}{$\begin{array}{l}\text { * Twice the area between the Lorenz curve and the diagonal } \\
\text { Averaged with the population prevalences of each exposure status as weights } \\
{ }^{\ddagger} \text { Mean absolute difference of the disease risks and/or relative risks of two randomly } \\
\text { selected subjects from the population } \\
\text { § Probability that the exposure level of a randomly selected case is heavier than that of } \\
\text { a randomly selected subject from the population } \\
\text { "Proportion of cases expected to be eliminated if an 'exposure-lowering' programme is } \\
\text { applied }\end{array}$} \\
\hline
\end{tabular}

and thus is invariant to scale changes. This property is essential to our purpose. For example, the degree of risk variation according to the levels of a particular exposure may be similar in two populations, but due to some 'population-specific' factors, the background risk of one of the populations may be much higher than the other. In this situation, the risk variation in the two populations will be rated the same by the index of coefficient of variation but not by the others. To derive such an index for our examples, we first calculate the 'means' of disease risk (0.00304, 0.00608 and 0.0152, respectively, see Table IV), either by weighted-averaging of the disease risks in each exposure level or simply by dividing the total case number by the total population number. Next, instead of calculating the 'standard deviation' of the disease risk, we calculate the 'mean differences' of the disease risk defined as the mean value of the absolute difference of the disease risks of two subjects, who are randomly selected one after another from the population with replacement. The 'mean difference' plays much the same role as the 'standard deviation', since the latter is just the 'root mean square deviation'. The mean differences of disease risk for the three examples are calculated as $0 \cdot 00179,0.00496$ and 0.00453 , respectively (Table IV). We then substitute the mean difference for the standard deviation in the formula for the coefficient of variation. The result is nothing but a similar index of dispersion/variation, but to avoid confusion, we refer to it as the 'coefficient of deviation'. The coefficients of deviation for the examples are $0.587,0.816$, and 0.298 , respectively. Therefore we see that the Gini index is equal to half of the 'coefficient of deviation' in disease risk.

(ii) 'Coefficient of deviation' in relative risk. From Table IV, we can check that the Gini index is equal to half of the coefficient of deviation in 'relative risk' as well. Although in the examples the relative risks are calculated relative to the lowest exposure level of 'none', the above-mentioned relation is actually invariant to the particular level of exposure taken as the reference for the relative risks. This again demonstrates the advantage of using the coefficient of deviation (and hence the Gini index) as an index of risk variation. 
(iii) 'Information content' of the exposure. It is of interest to find (see Table IV) that half of the Gini index plus $1 / 2$ can be shown equal to the probability that the exposure level of a randomly selected case is greater than that of a randomly selected subject from the population (note that if the selected pair is exposed the same, we toss a coin to decide whether the comparison is deemed 'greater'). Such a probability can be regarded as the 'information content' of the exposure. The higher the probability, the better we can discriminate the diseased from the ordinary. To be precise, consider a randomly selected pair of case and ordinary subject presented together with their exposure levels, but with the disease status being deliberately made blind. It is of interest to know how well the exposure information can 'recover' the disease status. The identification of the case with the heavier exposure level seems a reasonable strategy. The probability of a successful 'recovery' of the disease status for this strategy is equal to Gini/ $2+1 / 2$ and is a measure of information content of the exposure.

(iv) Impact fraction of an 'exposure-lowering' programme. Consider a situation where the exposure status of the individual subjects in a population are not fixed forever but are changing dynamically or stochastically - with transition probabilities equal to the population prevalences of each exposure level. Therefore, the exposure status of an individual may change but the population prevalences remain fixed. The concept of such a 'dynamically exposed' population can be a realistic description for some common exposures. For example, heavy smokers may abstain completely from smoking as time goes by, or the other way around, may indulge more. The residents living in a community may have a different degree of exposure to electromagnetic fields depending on, for example, the distances between their dwellings and the nearest power transmission lines, but in reality, people do not reside in the same place for ever but tend to move, and this represents yet another example of 'dynamic exposure'. Now, consider a public health intervention programme aiming not at completely removing the exposure but at 'lowering' it. The interpretation of 'lowering', in the first example, is that smokers are encouraged to abstain, completely or partially, from their smoking habit but once they show signs of greater indulgence, the intervention programme will take effect and maintain the smoking habit at their current levels. For the second example, with the 'exposure-lowering' programme implemented, people will move to a 'safer' place or else will not move at all. For a numerical illustration, return to the example in Table I. We trace the exposure changes of the 46,000 subjects in the 'medium' level. If the 'exposure-lowering' programme is not implemented, there will be $9200(46,000 \times 20$ per cent $)$ subjects completely unexposed, and $13,800(46,000 \times 30$ per cent $), 21,160(46,000 \times 46$ per cent $), 1380(46,000 \times 3$ per cent $)$, and $460(46,000 \times 1$ per cent $)$ subjects switch to 'light', 'medium', 'heavy' and 'extra-heavy' exposure levels, respectively. However, with the 'exposure-lowering' programme imposed, those who change to 'heavy' and 'extra-heavy' in the previous calculation will now be fixed at the original 'medium' level. Thus now we have 9200 unexposed, 13,800 light-exposed, and 23,000 $(21,160+1380+460)$ medium-exposed. The exposure changes for other exposure levels can be similarly calculated. Once the exposure distribution modified by the exposure-lowering programme has been obtained, we can calculate the proportion of cases expected to be eliminated. Though a 'target level' is not defined in this kind of intervention programme, we still refer to such proportions as the 'impact fraction' of the exposurelowering programme. The calculations show that these indices turn out interestingly to be just the corresponding Gini indices for the three examples in Tables I to III (see Table IV).

(v) 'Averaged' impact fraction. From Table IV, we can see that the Gini index is equal exactly to the 'averaged' impact fraction as well, which is defined in this paper as the weighted 
average of the impact fractions with weights taken as the population prevalences of each exposure status. In other words, the Gini index can be viewed as a summary index of the impact fractions. The idea of taking the average seems reasonable, at least when the 'target level' of an intervention programme is not or cannot be clearly defined. Although interpretation in terms of such naive averaging of impact fractions seems useless for field epidemiologists or public health professionals, it helps to make the Gini index even more transparent.

It should be pointed out that the relations between the Gini index and the various indices in the three examples are not a happy coincidence. Rather, the relations exist in general and can easily be derived using simple algebra (see Appendix). Also, since the Gini index can be interpreted by its relations with the aforementioned indices, each of which measures a different aspect of exposuredisease association, it is therefore no wonder that the ranking of the exposure impacts in the three examples in Tables I to III by such an index (examples in Table II $\gg$ Table I $\gg$ Table III, see Table IV) is consistent with our prior belief, while with the traditionally defined attributable risk, it is not.

\section{DISCUSSION}

To characterize exposure-disease association in human populations, epidemiologists have relied upon such indices as 'relative risk' and/or 'attributable risk' for quite a long time. This is probably because the message conveyed in the words 'relative' and/or 'attributable' is easily understandable and is self-evident even without resort to their formal definitions. However, when confronted by polytomous or continuous exposures, troubles then emerge, since, first of all, the index of relative risks is not in a common unit for comparison across different exposures or different diseases, and, secondly, the index of attributable risk sometimes has difficulty in selecting a suitable or realistic 'target level' and may not adequately catch and describe the variation of disease risks in populations. In this respect, the summary index of the Lorenz curve, the Gini index, with its desirable properties, seems a promising alternative as a fundamental measure in epidemiology.

The superiority of the Gini index seems obvious from a statistical point of view. If disease risks are not identical across different levels of an exposure variable in the population, the measures of the impact of this particular exposure containing only information about the mean value (the first moment) but not the variance (the second moment) will inherently lead to invalid inferences. Whereas the Gini index discussed in this paper is based on both the first (the weighted mean) and the second moment (the mean absolute difference) of the disease risks. Therefore it inherently contains more information and can better characterize the exposure-disease association.

In this paper, the examples we use to demonstrate the new methodology are exposures on a polytomous scale. However, the basic principle is the same for continuous or binary exposures. For the former, the Lorenz curve is similarly constructed by plotting the empirical cumulative distribution of the diseased against that of the general population, provided that the risk of disease is monotonically increasing as the exposure level increases. If this is not the case, we can categorize the data, re-arrange it from the lowest risk to the highest, and then use the method for polytomous exposure to construct (approximate) the respective Lorenz curve. For the binary case, it is clear that the corresponding Lorenz curve is composed of two straight lines. The Gini index in this case is twice the area of the triangle bound by these two lines and the diagonal and can be shown to be equal to the product of the unexposed proportion in the population and the traditionally defined attributable risk. It should be pointed out that the relations between the Gini index and the various indices described in this paper (the coefficients of deviation in disease risk or relative risk, the information content of the exposure, the impact fraction of an exposure- 
lowering programme, and the averaged impact fraction) still hold for continuous and binary exposures (we omit the proof). Consequently, the Gini index can be similarly interpreted. However, in the binary case, there seems no need to resort to the Gini index for the purpose of characterizing exposure-disease association, since the 'relative risk' now is a single-valued index which permits a direct comparison across different exposures and/or different diseases and the 'attributable risk' now has an unambiguous and indisputable 'target level'.

Although the Gini index has some advantages, as shown in this paper, some statistical issues need to be investigated further before adopting the index for general epidemiological usage. These include, first of all, the examination of its sampling variability under various sampling schemes such as case-control designs (including the unmatched and the matched types) and cohort studies (with and without loss to follow-up). From such studies, the testing of hypotheses as well as the construction of confidence intervals regarding such an index are then possible. Secondly, it is warranted to develop techniques for the calculation of the Gini index in general multivariate settings. Consequently, it is then possible to derive an 'adjusted' Gini index which takes into account the confounding effect of other risk factors. All these topics are, however, beyond the scope of this paper.

\section{APPENDIX}

Assume that the exposure under concern has $K$ levels which have been rearranged according to the disease risks (from the lowest to the highest) and are indexed by $i,(i=1,2, \ldots, K)$. The number of subjects in each exposure level is represented by $n_{i}$ and the number of cases by $d_{i}$. We let $N$ denote the total number of subjects in the population $\left(N=\sum_{i=1}^{K} n_{i}\right)$ and $D$ the total number of cases $\left(D=\sum_{i=1}^{K} d_{i}\right)$. The co-ordinates of the points in the Lorenz curve are denoted by $\left(x_{i}, y_{i}\right)$, $i=0,1,2, \ldots, K$. By definition, we have $x_{i}=\sum_{j \leqslant i} n_{j} / N$ and $y_{i}=\sum_{j \leqslant i} d_{j} / D$. Note that $\left(x_{0}, y_{0}\right)$ $=(0,0)$ and $\left(x_{K}, y_{K}\right)=(1,1)$.

The Gini index can be calculated in two ways. The first involves direct calculation of the area between the Lorenz curve and the diagonal, which yields:

$$
\text { Gini }=\sum_{i=1}^{K}\left|\begin{array}{ll}
x_{i-1} & y_{i-1} \\
x_{i} & y_{i}
\end{array}\right|=\sum_{i=1}^{K}\left(x_{i-1} y_{i}-x_{i} y_{i-1}\right) .
$$

The second makes use of the relation 'the area under the Lorenz curve' $=1 / 2-$ Gini $/ 2=\sum_{i=1}^{K}\left(x_{i}-x_{i-1}\right)\left(y_{i}+y_{i-1}\right) / 2$. Next, we demonstrate the relation of the Gini index to the other various indices.

(i) Coefficient of deviation in disease risk

$$
\begin{aligned}
& =\frac{\sum_{i=1}^{K} \sum_{j=1}^{K} \frac{n_{i}}{N} \frac{n_{j}}{N}\left|\frac{d_{i}}{n_{i}}-\frac{d_{j}}{n_{j}}\right|}{\frac{D}{N}} \\
& =\sum_{i=1}^{K}\left[\sum_{j \leqslant i}\left(\frac{n_{j}}{N} \frac{d_{i}}{D}-\frac{n_{i}}{N} \frac{d_{j}}{D}\right)+\sum_{j>i}\left(\frac{n_{i}}{N} \frac{d_{j}}{D}-\frac{n_{j}}{N} \frac{d_{i}}{D}\right)\right] \\
& =\sum_{i=1}^{K}\left[\left(y_{i}-y_{i-1}\right) x_{i}-\left(x_{i}-x_{i-1}\right) y_{i}+\left(x_{i}-x_{i-1}\right)\left(1-y_{i}\right)-\left(y_{i}-y_{i-1}\right)\left(1-x_{i}\right)\right] \\
& =\sum_{i=1}^{K}\left(x_{i}-x_{i-1}\right)-\sum_{i=1}^{K}\left(y_{i}-y_{i-1}\right)+2 \sum_{i=1}^{K}\left(x_{i-1} y_{i}-x_{i} y_{i-1}\right)=2 \text { Gini. }
\end{aligned}
$$


(ii) The relation between the coefficient of deviation in relative risk and the Gini index can be similarly derived as in (i).

(iii) Information content of the exposure

$$
\begin{aligned}
& =\sum_{i=1}^{K} \frac{n_{i}}{N}\left(\frac{1}{2} \frac{d_{i}}{D}+\sum_{j>1} \frac{d_{j}}{D}\right)=\sum_{i=1}^{K}\left(x_{i}-x_{i-1}\right)\left[\frac{\left(y_{i}-y_{i-1}\right)}{2}+\left(1-y_{i}\right)\right] \\
& =\sum_{i=1}^{K}\left(x_{i}-x_{i-1}\right)\left[1-\frac{\left(y_{i}+y_{i-1}\right)}{2}\right]=\sum_{i=1}^{K}\left(x_{i}-x_{i-1}\right)-\sum_{i=1}^{K}\left(x_{i}-x_{i-1}\right) \frac{\left(y_{i}+y_{i-1}\right)}{2} \\
& =\frac{1}{2}+\frac{\text { Gini }}{2}
\end{aligned}
$$

(iv) Impact fraction of the exposure-lowering programme

$$
\begin{aligned}
& =\frac{1}{D} \sum_{i=1}^{K}\left[d_{i}-n_{i}\left(\sum_{j<i} \frac{n_{j}}{N} \frac{d_{j}}{n_{j}}+\sum_{j \geqslant i} \frac{n_{j}}{N} \frac{d_{i}}{n_{i}}\right)\right] \\
& =\sum_{i=1}^{K}\left(\frac{d_{i}}{D}-\frac{d_{i}}{D} \sum_{j \geqslant i} \frac{n_{j}}{N}-\frac{n_{i}}{N} \sum_{j<i} \frac{d_{j}}{D}\right) \\
& =\sum_{i=1}^{K}\left[\left(y_{i}-y_{i-1}\right) x_{i-1}-\left(x_{i}-x_{i-1}\right) y_{i-1}\right]=\sum_{i=1}^{K}\left(x_{i-1} y_{i}-x_{i} y_{i-1}\right)=\text { Gini. }
\end{aligned}
$$

(v) Averaged impact fraction

$$
\begin{aligned}
& =\sum_{i=1}^{K} \frac{n_{i}}{N} \frac{\sum_{j>i} d_{j}-\frac{d_{i}}{n_{i}} \sum_{j>i} n_{j}}{D}=\sum_{i=1}^{K}\left(\frac{n_{i}}{N} \sum_{j>i} \frac{d_{j}}{D}-\frac{d_{i}}{D} \sum_{j>i} \frac{n_{j}}{N}\right) \\
& =\sum_{i=1}^{K}\left[\left(x_{i}-x_{i-1}\right)\left(1-y_{i}\right)-\left(y_{i}-y_{i-1}\right)\left(1-x_{i}\right)\right] \\
& =\sum_{i=1}^{K}\left(x_{i}-x_{i-1}\right)-\sum_{i=1}^{K}\left(y_{i}-y_{i-1}\right)+\sum_{i=1}^{K}\left(x_{i-1} y_{i}-x_{i} y_{i-1}\right)=\text { Gini. }
\end{aligned}
$$

\section{REFERENCES}

1. Breslow, N. E. and Day, N. E. Statistical Methods in Cancer Research, Vol I, IARC Scientific Publication No. 32, Lyon, 1980.

2. Cornfield, J., Haenszel, W., Hammond, E. C., Lilienfeld, A. M., Shimkin, M. B. and Wynder, E. L. 'Smoking and lung cancer: recent evidence and a discussion of some questions', Journal of the National Cancer Institute, 22, 173-203 (1959).

3. Rothman, K. J. Modern Epidemiology, Little, Brown and Company, Boston, 1986.

4. Lilienfeld, A. M. and Lilienfeld, D. E. Foundations of Epidemiology, 2nd edn, Oxford University Press, New York, 1980.

5. Cole, P. and MacMahon, B. 'Attributable risk percent in case-control studies', British Journal of Preventive and Social Medicine, 25, 242-244 (1971).

6. Miettinen, O. S. 'Proportion of disease caused or prevented by a given exposure, trait or intervention', American Journal of Epidemiology, 99, 325-332 (1974).

7. Levin, M. L. 'The occurrence of lung cancer in man', Acta Union Internationalis contra Cancrum, $\mathbf{9}$, 531-541 (1953).

8. Morgenstern, H. and Bursic, E. S. 'A method for using epidemiologic data to estimate the potential impact of an intervention on the health status of a target population', Journal of Community Health, 7 , 292-309 (1982). 
9. Walter, S. D. 'The estimation and interpretation of attributable risk in health research', Biometrics, 32, 829-849 (1976).

10. Greenland, S. and Drescher, K. 'Maximum likelihood estimation of the attributable fraction from logistic models', Biometrics, 49, 865-872 (1993).

11. Ekelund, R. B. and Tollison, R. D. Economics, Little, Brown and Company, Boston, 1986.

12. Shryock, H. S. and Siegel, J. S. The Methods and Materials of Demography, U.S. Government Printing Office, Washington, 1975.

13. Lee, W. C. 'Analysis of seasonal data using the Lorenz curve and the associated Gini index', International Journal of Epidemiology, 25, 426-434 (1996). 